\title{
EXPLORACIÓN CON REDES NEURONALES ARTIFICIALES PARA ESTIMAR LA RESISTENCIA A LA COMPRESIÓN, EN CONCRETOS FIBROREFORZADOS CON ACERO
}

\author{
EXPLORING ARTIFICIAL NEURAL NETWORKS TO ESTIMATE COMPRESSIVE STRENGTH OF STEEL \\ FIBER-REINFORCED CONCRETE \\ Luis Octavio González Salcedo
}

Ing. Civil, M.Sc., Profesor Asociado, Departamento de Ingeniería, Grupo de Investigación en Materiales y Medio Ambiente.

Facultad de Ingeniería y Administración, Universidad Nacional de Colombia sede Palmira;

Grupo de Materiales Compuestos, Facultad de Ingeniería,

Universidad del Valle, Cali, Colombia,

logonzalezsa@unal.edu.co

Aydée Patricia Guerrero Zúñiga

Ing. Civil, MSc., Ph.D., Profesora Titular, Escuela de Geomática e Ingeniería Civil, Grupo de Investigación en Ingeniería Sísmica, Ingeniería Eólica y Estructuras Inteligentes,

Facultad de Ingeniería, Universidad del Valle, Cali, Colombia,

aydeegzu@univalle.edu.co

Silvio Delvasto Arjona

Ing. Químico, MSc., Ph.D., Profesor Titular, Escuela de Ingeniería de Materiales,

Grupo de Materiales Compuestos, Facultad de Ingeniería,

Universidad del Valle, Cali, Colombia,

silviodelvasto@hotmail.com

Adrián Luis Ernesto Will

Matemático, Ph.D., Departamento de Matemáticas, Facultad de Ciencias Exactas y Tecnología, Universidad Nacional de Tucumán; Centro de Investigación en Tecnologías Avanzadas de

Tucumán, Universidad Tecnología Nacional - Facultad Regional Tucumán,

San Miguel de Tucumán, Argentina,

awill@herrera.unt.edu.ar, awill@citat.org.ar

Fecha de recepción: 23 de febrero de 2012

Fecha de aprobación: 23 de mayo de 2012

\section{RESUMEN}

En diseño y construcción de estructuras de concreto, la resistencia a la compresión a 28 días de curado es la especificación de control de estabilidad de la obra. La inclusión de fibras como reforzamiento 
de la matriz cementicia permite una ganancia en sus propiedades, además de obtener un material de alto desempeño. En las normativas, se plantean formulaciones predictivas de la resistencia a la compresión basadas en unos pocos parámetros de composición del concreto, tales como la relación agua/cemento y el contenido de cemento Portland. Por otra parte, también se han planteado métodos de diseños de concreto para definir la ponderación de sus materiales componentes, teniendo como referencia la resistencia a la compresión del concreto simple. Además, las redes neuronales artificiales, como un símil de las neuronas biológicas, han sido utilizadas como herramientas de predicción de la resistencia a la compresión en el concreto, también con referencia al concreto simple, sin reforzamiento con fibras. Los antecedentes en este uso muestran que es interesante desarrollar aplicaciones en los concretos reforzados con fibras. En el presente trabajo se elaboraron redes neuronales artificiales para predecir la resistencia a la compresión en concretos reforzados con fibras de acero. Los resultados de los indicadores de desempeño mostraron que las redes neuronales artificiales elaboradas pueden realizar una aproximación adecuada al valor real de la propiedad mecánica.

Palabras clave: resistencia a la compresión; concreto reforzado con fibras, fibra de acero, predicción, inteligencia artificial, redes neuronales artificiales.

\begin{abstract}
By designing and building concrete structures, the compressive strength achieved at 28-day curing typically represents the stability control specification of any work. Furthermore, reinforcing fibers into the cement based matrix has allowed a gain to their properties, as well as a high performance material. Technical literature states predictive formulations of compressive strength of concrete in function of a few composition parameters, such as water/cement ratio and the Portland cement. Also, there are formulations to find the proportion of the raw materials to get a defined compressive strength, specifically non-reinforced ordinary concrete. Besides artificial neural networks as a metaphor of biological neurons have been used as a tool to predict concrete compressive strength. The experience in this application shows an increasing interest to develop applications using fiber-reinforced concrete. In this paper, an artificial neural network has been developed to predict the compressive strength of steel-fiber-reinforced-concrete. The results prove that developed artificial neural networks may perform an adequate approximation to the actual value of the mechanical property.
\end{abstract}

Keywords: compressive strength, fiber-reinforced concrete, steel fiber, prediction, artificial intelligence, artificial neural networks.

\title{
INTRODUCCIÓN
}

El concreto es uno de los más importantes materiales de ingeniería, usado en la construcción de edificaciones, puentes, túneles y otras estructuras [1]. El concreto es un conglomerado elaborado 
a partir de agregados (finos y gruesos), y cemento con una adecuada y controlada cantidad de agua, así como de otros componentes denominados adiciones minerales (humo de sílice, cenizas volantes, escorias y otros materiales puzolánicos), y aditivos químicos (reductores de agua y agentes inclusores de aire, entre otros) [2]. Una de las propiedades mecánicas que se usa en el concreto, es la resistencia a la compresión que alcanza en un período de curado de 28 días [1].

La curva esfuerzo-deformación unitaria del concreto, muestra que éste es un material frágil; la incorporación de fibras como reforzamiento de la matriz cementicia, ha permitido extender el área de dicha curva más allá de la aparición de la primera grieta y seguir soportando esfuerzos, a pesar de haber alcanzado su máxima resistencia [3]. Esto se conoce como un comportamiento cuasi - dúctil que le otorga al material, propiedades adicionales como control de grietas, mayor durabilidad a agentes corrosivos, adecuado comportamiento en áreas sometidas a vibración, entre otras, lo plantea Ahmed S.F.U., y Mihashi H. [4].

En el concreto, la resistencia a la compresión está influenciada además de las proporciones de la mezcla, por la calidad de sus diferentes ingredientes, las condiciones de curado, la relación agua/ cemento, y los métodos de mezclado, transporte, colocación y vibración [1]. La importancia de la predicción de la resistencia de diseño del concreto antes de los 28 días, ha sido reconocida en la actual construcción con este material y en el juicio moderno de la ingeniería. Por tal razón, métodos convencionales basados en desarrollos estadísticos, usando ecuaciones de regresiones lineales y no lineales, han sido construidos para modelar el problema de la predicción, en los cuales la alta dependencia no lineal entre sus factores o variables influyentes en la propiedad mecánica, no ha sido generalmente considerada [5-8].

Esta complejidad conlleva a estimar la resistencia a la compresión del concreto por medio de la Inteligencia Artificial, que reúne una serie de técnicas informáticas para realizar funciones de aprendizaje y autocorrección mediante algoritmos o códigos de programación computacional para resolver diversos problemas, de forma similar a como lo haría el ser humano [9]. Algunos de los principales paradigmas de la inteligencia artificial entre muchos otros, son las redes neuronales artificiales, los algoritmos evolutivos y la lógica difusa.

En el campo de la modelación, las redes neuronales artificiales (RNA), son modelos de caja negra o model-free estimators, desarrollados para resolver problemas en los cuales las relaciones de los diferentes componentes son complejas, las variables o reglas de relación no son fáciles de obtener, hay escaso conocimiento, pero sí existe la experiencia de una serie de datos [10]. Estas redes, también son referidas como redes neuronales, neuro-computacionales, redes conectadas, procesadores paralelamente distribuidos, etc.; son sistemas inteligentes inspirados en los sistemas neurales biológicos. Desde el punto de vista funcional, son procesadores de información con un canal de entrada de información y un canal de salida, con gran capacidad de comunicar y unirse entre sí, y su unión se denomina sinapsis. 
Existen diversos estudios antecedentes del uso de RNA para estimar la resistencia a la compresión en concretos no reforzados con fibras [11-17], donde las variables de entrada se han enfocado en las cantidades de los componentes de la mezcla, usando la técnica mencionada en concretos reforzados con fibras. En el presente trabajo, se exploró el uso de RNA en la estimación de la resistencia de diseño a la resistencia a la compresión en concretos reforzados con fibras de acero, y se considera la dosificación de la mezcla, y otras características propias de sus componentes.

\section{MATERIALES Y MÉTODOS}

\subsection{REVISIÓN DE FUNDAMENTOS TEÓRICOS}

Redes Neuronales Artificiales. La RNA es un modelo matemático que emula el sistema neuronal biológico en el proceso de la información, y en cuyo símil, la integración de las neuronas artificiales se realiza mediante funciones matemáticas que procesan y envían información entre sí. La información que se transmite a través de las conexiones de la red, se ponderó en pesos de importancia, para modular así la intensidad de la relación entre neuronas. La fundamentación matemática para estructurar una RNA, está basada en el Teorema de Kolmogorov [18, 19], de tal forma que a partir de tres capas (de entrada, oculta y de salida), se puede aproximar cualquier función continua hasta el nivel deseado [20]. El uso de dos o más capas ocultas hace más operativa la red porque permite flexibilizar la elección de las funciones de transferencia o disminuir el número de neuronas [21].

La elaboración de una RNA involucra definir el número de capas ocultas y el número de neuronas en ellas, el tipo de conexiones entre neuronas, la función de transferencia o respuesta de las neuronas y el mecanismo de aprendizaje. Con respecto del tipo de conexiones, una tipología es la feedforward definida por Rumelhart D.E., et al. [22], la cual corresponde a un tipo de estructura de computación paralela donde muchas pequeñas unidades de cálculo llamadas neuronas, están masivamente interconectadas con la capa anterior de donde reciben información, y con la capa posterior hacia donde la transmiten. Sus capas ocultas poseen una función de activación que limita la salida a un rango cercano y desde esta la capa de salida, puede producir todos los valores de estimación. La salida de cada capa está representada en la ecuación 1 como:

$$
\mathrm{Y}_{\mathrm{N} \times 1}=\mathrm{f}(\mathrm{Z})=\mathrm{f}\left(\mathrm{W}_{\mathrm{N} \times \mathrm{M}} \mathrm{X}_{\mathrm{M}, 1}+\mathrm{b}_{\mathrm{N}, 1}\right)
$$

Donde, $Y$ es un vector que contiene la salida desde cada una de las $N$ neuronas en una capa dada, $W$ es la matriz que contiene los pesos sinápticos (importancia), para cada una de las $M$ salidas para todas las $N$ neuronas, $X$ es el vector que contiene las entradas, $b$ es el vector que contiene los sesgos (biases), y fes la función de activación. La función de activación no-lineal corresponde a una función sigmoidea que se expresa en la ecuación 2: 


$$
g(Z)=\frac{1}{1+e^{-z}}
$$

Siendo $Z$ la expresión dentro del paréntesis de la ecuación 1. La cantidad mínima de neuronas de las capas ocultas puede obtenerse entre los valores conseguidos a partir de la regla de la pirámide [23, 24], ecuación 3, y la aplicación del Teorema de Kolmogorov [21], ecuación 4:

$$
\begin{gathered}
k=\operatorname{Redondeo~}(\sqrt{ } \mathrm{n} \mathrm{m}) \\
k=2 n+1
\end{gathered}
$$

Donde, $k$ es el número de neuronas de la capa oculta, $n$ es el número de neuronas de la capa de entrada (variables de entrada), y $m$ es el número de neuronas de la capa de salida (variables de la capa de salida). Un procedimiento muy usado es iniciar una red de prueba con el número mínimo de neuronas ocultas, y si los resultados no son satisfactorios, se va aumentando progresivamente su número hasta que el indicador de evaluación sea aceptable [25].

La técnica de aprendizaje de backpropagation consiste en utilizar una optimización basada en derivadas, donde el error es derivable no sólo en función de los pesos de la capa de salida, sino también en función de los pesos de la capa oculta, utilizando la regla de la cadena definida en Hinton G. [25], que permite minimizar el error de entrenamiento de la red neuronal, que es la diferencia entre los valores calculados por la red neuronal, utilizando un determinado conjunto de pesos y los valores originales. El error y las correcciones efectuadas a los pesos, se trasladan desde la capa de salida hacia atrás hasta la capa de entrada, de donde surge su nombre de propagación hacia atrás. El método de descenso por gradiente o gradiente conjugado, correspondiente al gradiente conjugado con escalamiento SCG (Scale Conjugate Gradient) [26], sustituye la búsqueda lineal por un escalamiento de paso, que depende del éxito en la reducción del error y buen desempeño de la aproximación cuadrática. El método de entrenamiento denominado Algoritmo de Levenberg-Mardquardt LMA (Levenberg-Marquardt Algorithm) [27], corresponde a una técnica iterativa que localiza el mínimo de una función que se expresa como la suma de los cuadrados de funciones no lineales.

Para reconocer patrones, la RNA divide la información en tres grupos, uno de los cuales es una técnica de división, $K$-Fold Cross Validation con $k=3$, que divide cada base de datos específica en tres grupos de igual extensión con vectores de información escogidos al azar, usando alternativamente dos grupos como conjunto de educación de la red (entrenamiento y prueba), y el tercero como validación [28]. Esta técnica utiliza también, una regulación estadística bayesiana, elimina los sesgos de elección y entrega una mejor idea de los errores de validación y de la existencia de valores extremos o atípicos (outliers), que normalmente quedan ocultos por la división de datos y otros efectos [28]. 
Dependencia de la resistencia a la compresión. La resistencia a la compresión de los concretos, depende de la resistencia del mortero (mezcla de cemento, agua y arena), de los efectos que sobre la mezcla tengan el agregado grueso y la interfase entre estos dos compuestos, del contenido de aire y de las adiciones minerales. Dentro de las variables influyentes de la resistencia del mortero, se consideran los contenidos de cemento y agua, y el tipo de cemento [29].

El comportamiento de la zona de transición es influenciada además de la cantidad, por las características de las partículas del agregado tales como el tamaño, la forma, la textura de la superficie y el tipo de mineral [29-33]. El efecto del contenido del aire es influenciado por la cantidad del agente inclusor de aire [29]. Las cantidades de las adiciones de humo de sílice, de cenizas volantes y de escoria de alto horno, cuando son utilizadas como materiales cementantes en reemplazo de parte del cemento, influyen en la resistencia a la compresión [29], al igual que los agentes reductores de agua [34].

Las propiedades del concreto reforzado con fibras, están relacionadas con las propiedades de la matriz, de las fibras y de la interfase entre los dos componentes, donde se resalta el volumen de fibra incorporado en la matriz cementicia y un parámetro de adherencia de la fibra [35]. Hay CERCA de 11 diferentes tipos de fibra disponibles comercialmente [35, 36], Figura 1. La ASTM A 820 provee una clasificación en cuatro tipos, de acuerdo con su fabricación y la JSCE las clasifica en tres grupos de acuerdo con la forma de su sección transversal [35].

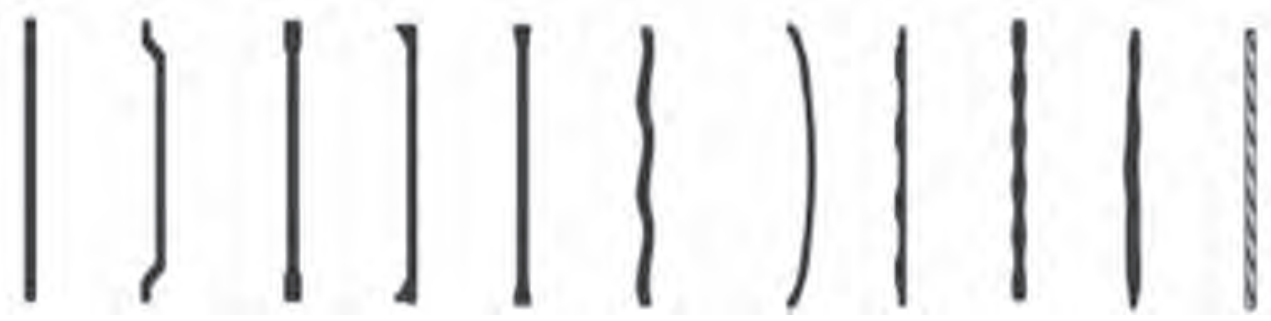

Figura 1. Tipos de fibras de acero, comercialmente disponibles [36] y [37]. De izquierda a derecha, de acuerdo con los nombres conocidos comercialmente: rectas (lisas), extremos en ganchos, extremos en palas, extremos en perillas, extremos cónicos, onduladas (corrugadas), en forma de arcos, dentadas, superficie indentada, irregulares y torsionadas.

\subsection{BASE DE DATOS PARA ENTRENAMIENTO DE LA RNA}

Se usó una base de datos elaborada a partir de una amplia información disponible sobre resultados experimentales de ensayos de resistencia a la compresión para diversos diseños de mezclas, reportados en publicaciones diversas [36-94]. La base de datos se utilizó para enseñanza, entrenamiento y validación computacional de las redes neuronales artificiales, se encuentra conformada por 323 registros que constituyen los vectores de información completa del arreglo matricial de la base de datos para las variables involucradas. La resistencia de diseño a la compresión presentada en los reportes originales está basada en la metodología del ensayo estandarizado ASTM C39[1, 95]. 
Para seleccionar las variables de entrada y conformación de los vectores de información (registros de información), se tuvo en cuenta las consideraciones sobre la dependencia de la resistencia a la compresión en concretos reforzados con fibras de acero. Para representar la influencia de la forma y la textura de la superficie, se diferenció entre agregado triturado y de canto rodado. Para el parámetro del agregado grueso, clasificado en tres grupos, de acuerdo con la afectación sobre las propiedades mecánicas del concreto, se adaptaron los tipos litológicos para representar la procedencia del agregado [96, 99].

Las 20 variables contenidas en la base de datos, se agruparon en la Tabla 1 así: cemento, adiciones minerales, agua total, agregados, aire incluido, fibra de acero, y resistencia de diseño a la compresión. Esta última corresponde a la variable de salida.

Tabla 1. Detalles de las variables incorporadas en la base de datos para el entrenamiento de la Red Neuronal Artificial, con información de mezclas de concreto reforzadas con fibras de acero

\begin{tabular}{|c|c|c|c|c|c|}
\hline GRUPO & CONCEPTO & VARIABLE & UNIDAD & SíMBOLO & $\begin{array}{l}\text { REPRESENTACIÓN EN LA } \\
\text { BASE DE DATOS }\end{array}$ \\
\hline & & Cantidad & $\mathrm{Kg} / \mathrm{m}^{3}$ & C & Numérica real \\
\hline Cementos & Cemento & $\begin{array}{l}\text { Tipo I } \\
\text { Tipo II } \\
\text { Tipo III } \\
\text { Tipo IV } \\
\text { Tipo V }\end{array}$ & - & $\begin{array}{l}C-1 \\
C-2 \\
C-3 \\
C-4 \\
C-5\end{array}$ & $\begin{array}{l}{\left[\begin{array}{lllll}1 & 0 & 0 & 0 & 0\end{array}\right]^{*}} \\
{\left[\begin{array}{llllll}0 & 1 & 0 & 0 & 0\end{array}\right]^{*}} \\
{\left[\begin{array}{lllll}0 & 0 & 1 & 0 & 0\end{array}\right]^{*}} \\
{\left[\begin{array}{llllll}0 & 0 & 0 & 1 & 0\end{array}\right]^{*}} \\
{\left[\begin{array}{llllll}0 & 0 & 0 & 0 & 1\end{array}\right]^{*}}\end{array}$ \\
\hline \multirow{3}{*}{ Adiciones } & Humo de Sílice & Cantidad & $\mathrm{Kg} / \mathrm{m}^{3}$ & SF & Numérica real \\
\hline & Cenizas volantes & Cantidad & $\mathrm{Kg} / \mathrm{m}^{3}$ & $\mathrm{FA}$ & Numérica real \\
\hline & Escoria & Cantidad & $\mathrm{Kg} / \mathrm{m}^{3}$ & GBFS & Numérica real \\
\hline \multirow[b]{3}{*}{ Agua total } & Agua & Cantidad & $\mathrm{Kg} / \mathrm{m}^{3}$ & W & Numérica real \\
\hline & & Cantidad & $\mathrm{Kg} / \mathrm{m}^{3}$ & HWR & Numérica real \\
\hline & Agente reductor & $\begin{array}{c}\text { Sin aditivo } \\
\text { Plastificante } \\
\text { Superplastificante } \\
\end{array}$ & - & $\begin{array}{l}\text { HWR-P } \\
\text { HWR-SP }\end{array}$ & $\begin{array}{l}{\left[\begin{array}{ll}0 & 0\end{array}\right]^{*}} \\
{\left[\begin{array}{ll}1 & 0\end{array}\right]^{*}} \\
{\left[\begin{array}{ll}0 & 1\end{array}\right]^{*}}\end{array}$ \\
\hline \multirow{6}{*}{ Agregados } & & Cantidad & $\mathrm{Kg} / \mathrm{m}^{3}$ & $S$ & Numérica real \\
\hline & Arena & $\begin{array}{c}\text { Lecho de río (canto } \\
\text { rodado) } \\
\text { Cantera (triturado) }\end{array}$ & - & $\begin{array}{l}\text { S-CR } \\
\text { S-TR }\end{array}$ & $\begin{array}{l}{\left[\begin{array}{ll}1 & 0\end{array}\right]^{\star}} \\
{\left[\begin{array}{ll}0 & 1\end{array}\right]^{\star}}\end{array}$ \\
\hline & \multirow{4}{*}{ Grava } & Cantidad & $\mathrm{Kg} / \mathrm{m}^{3}$ & G & Numérica real \\
\hline & & $\begin{array}{c}\text { Lecho de río (canto } \\
\text { rodado) } \\
\text { Cantera (triturado) }\end{array}$ & - & $\begin{array}{l}\text { G-CR } \\
\text { G-TR }\end{array}$ & $\begin{array}{l}{\left[\begin{array}{ll}1 & 0\end{array}\right]^{*}} \\
{\left[\begin{array}{ll}0 & 1\end{array}\right]^{*}}\end{array}$ \\
\hline & & Tamaño máximo & $\mathrm{mm}$ & G-TMA & Numérica real \\
\hline & & $\begin{array}{l}\text { Clase } 1 \\
\text { Clase } 2 \\
\text { Clase } 3\end{array}$ & - & $\begin{array}{l}\text { LTCA-1 } \\
\text { LTCA-2 } \\
\text { LTCA-3 }\end{array}$ & $\begin{array}{l}{\left[\begin{array}{lll}1 & 0 & 0\end{array}\right]^{*}} \\
{\left[\begin{array}{lll}0 & 1 & 0\end{array}\right]^{*}} \\
{\left[\begin{array}{lll}0 & 0 & 1\end{array}\right]^{*}}\end{array}$ \\
\hline
\end{tabular}




\begin{tabular}{|c|c|c|c|c|c|}
\hline GRUPO & CONCEPTO & VARIABLE & UNIDAD & SÍMBOLO & $\begin{array}{l}\text { REPRESENTACIÓN EN LA } \\
\text { BASE DE DATOS }\end{array}$ \\
\hline $\begin{array}{c}\text { Aire } \\
\text { incluido }\end{array}$ & Agente incluso & Cantidad & $\mathrm{Kg} / \mathrm{m}^{3}$ & AEA & Numérica real \\
\hline \multirow{4}{*}{ Fibra } & \multirow{4}{*}{ Fibra de acero } & Cantidad & $\mathrm{Kg} / \mathrm{m}^{3}$ & PPFR & Numérica real \\
\hline & & $\begin{array}{c}\text { Tipo y anclaje: } \\
\text { Sin fibra } \\
\text { Clase } 1 \\
\text { Clase } 2 \\
\text { Clase } 3 \\
\text { Clase } 4\end{array}$ & - & $\begin{array}{l}\text { SFR-1 } \\
\text { SFR-2 } \\
\text { SFR-3 } \\
\text { SFR-4 }\end{array}$ & $\begin{array}{l}{\left[\begin{array}{llll}0 & 0 & 0 & 0\end{array}\right]^{*}} \\
{\left[\begin{array}{llll}1 & 0 & 0 & 0\end{array}\right]^{*}} \\
{\left[\begin{array}{llll}0 & 1 & 0 & 0\end{array}\right]^{*}} \\
{\left[\begin{array}{llll}0 & 0 & 1 & 0\end{array}\right]^{*}} \\
{\left[\begin{array}{llll}0 & 0 & 0 & 1\end{array}\right]^{*}}\end{array}$ \\
\hline & & Longitud & $\mathrm{mm}$ & Lf & Numérica real \\
\hline & & Relación de aspecto & - & Lf/Df & Numérica real \\
\hline $\begin{array}{l}\text { Variable a } \\
\text { estimar }\end{array}$ & $\begin{array}{c}\text { Propiedad } \\
\text { mecánica a } \\
\text { estimar }\end{array}$ & $\begin{array}{c}\text { Resistencia de diseño } \\
\text { a la compresión }\end{array}$ & $\mathrm{MPa}$ & $f^{\prime} C$ & Numérica real \\
\hline
\end{tabular}

*Variables discretas o codificadas, y para cada caso, son mutuamente excluyentes.

\subsection{ELABORACIÓN DE LA RNA}

En el Laboratorio Computacional del Departamento de Matemáticas de la Universidad Nacional de Tucumán, se elaboró un conjunto de programas con el algoritmo para la RNA usada en la estimación de la resistencia a la compresión del concreto.

La información recopilada en la base de datos general, fue arreglada para conformar tres subconjuntos con 214, 312 y 323 vectores de información, respectivamente que a su vez, constituyeron tres nuevas bases de datos específicas y que relacionan como información de interés para su estimación, los valores de resistencia a la compresión del concreto (f'c). Como lenguaje de programación para la escritura del algoritmo de la RNA se usó Matlab ( R); la RNA se creó, usando la caja de herramientas de redes neuronales del lenguaje mencionado [100], los vectores de información fueron leídos directamente en el programa en una matriz creada para almacenar la base de datos. La tipología de la RNA usada corresponde a una red multicapa (feedforward), y se usó una metodología de educación o aprendizaje de propagación hacia atrás (backpropagation), se utilizó una técnica de división de datos para enseñanza-entrenamientovalidación denominada validación cruzada (K-Fold Cross Validation) con k = 3 . 
Tabla 2. Descripción de los módulos que conforman el conjunto de programas (principal y cinco auxiliares), para el entrenamiento de la RNA

\begin{tabular}{|l|l|}
\hline \multicolumn{1}{|c|}{$\begin{array}{c}\text { DENOMINACIÓN } \\
\text { GENÉRICA }\end{array}$} & \multicolumn{1}{c|}{ FUNCIÓN } \\
\hline Redes & $\begin{array}{l}\text { Carga y entrena las redes neuronales artificiales, y constituye el programa principal } \\
\text { para el entrenamiento de la RNA. }\end{array}$ \\
\hline Configurar variables & $\begin{array}{l}\text { Carga las variables y parámetros necesarios para correr Leave-One-Out-CrossValida- } \\
\text { tion de la RNA, con un procesamiento previo de los datos utilizados. }\end{array}$ \\
\hline Preparar datos & $\begin{array}{l}\text { Prepara los datos, realiza Leave-One-Out CrossValidation, hace la división de datos } \\
\text { de Entrenamiento y Validación, y carga los resultados en las variables globales. }\end{array}$ \\
\hline Validación cruzada & $\begin{array}{l}\text { Ejecuta la técnica de validación cruzada denominada Leave-One-Out-CrossValida- } \\
\text { tion. La técnica consiste en elegir un dato del conjunto de chequeo, y todos los } \\
\text { demás datos disponibles son seleccionados como conjunto de entrenamiento. Si el } \\
\text { error de predicción es grande, el dato es evaluado con respecto de su anormalidad, } \\
\text { es decir, si es muy distante de los demás, y es eliminado. }\end{array}$ \\
\hline Insertar red & $\begin{array}{l}\text { Diseñado como una herramienta para separar exactamente donde está la creación } \\
\text { de la RNA. Esta herramienta permite que con el mismo sistema, se pueda probar } \\
\text { en futuras aplicaciones otros tipos de redes. El objetivo del programa es construir y } \\
\text { entrenar la RNA. }\end{array}$ \\
\hline Entrenamiento & \begin{tabular}{l} 
Simula la RNA. \\
\hline
\end{tabular}
\end{tabular}

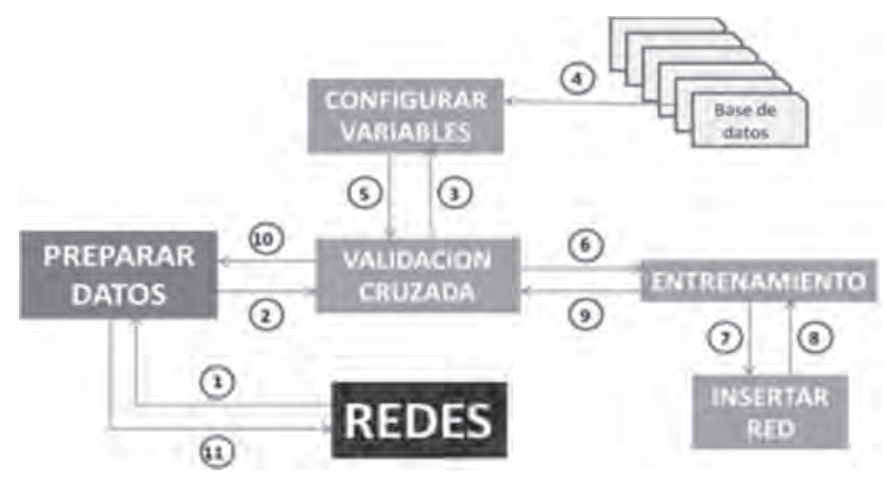

Figura 2. Esquema gráfico de interrelación de los módulos dentro del conjunto de programas (principal y cinco auxiliares), para entrenar una RNA de estimación de la resistencia a la compresión.

El conjunto de programas se estructuró con 6 módulos, y comprende un programa principal para entrenar la RNA y cinco programas auxiliares. En la Tabla 2, se hace una breve descripción de la función de cada módulo y en la Figura 2, se presenta un esquema gráfico de la interrelación de los módulos dentro del conjunto de programas. 


\subsection{ARQUITECTURA DE LA RNA}

En el presente trabajo, se ha configurado la RNA con una capa de entrada, dos capas ocultas y una capa de salida. La Tabla 3 muestra para el caso de estudio, las pruebas realizadas en una de las redes conformadas, para definir el número de neuronas en las capas ocultas, eligiéndose una red con dos capas ocultas de 50 y 10 neuronas, respectivamente. Para preparar los datos, se eligió como forma de entrenamiento el método de descenso por gradiente o gradiente conjugado, correspondiente al gradiente conjugado con escalamiento SCG. Una vez que se ejecutó el programa principal para la RNA, se eligió como método de entrenamiento el Algoritmo LMA.

Tabla 3. Prueba para la selección del número de neuronas en las dos capas ocultas, usando como indicador de evaluación el factor de correlación $\mathrm{R}^{2}$

\begin{tabular}{|c|c|c|c|c|}
\hline CONJUNTO & ENTRENAMIENTO & PRUEBA & VALIDACIÓN & TOTAL \\
\hline Arquitectura de la RNA* & $\mathbf{R}^{2}$ & $\mathbf{R}^{2}$ & $\mathbf{R}^{2}$ & $\mathbf{R}^{2}$ \\
\hline [05 05] & 1 & 0,7663 & 0,8524 & 0,9236 \\
\hline$\left[\begin{array}{ll}10 & 10\end{array}\right]$ & 1 & 0,5947 & 0,7979 & 0,7643 \\
\hline$\left[\begin{array}{lll}20 & 20\end{array}\right]$ & 1 & 0,7103 & 0,7643 & 0,9231 \\
\hline$\left[\begin{array}{lll}30 & 30\end{array}\right]$ & 1 & 0,5306 & 0,6644 & 0,8711 \\
\hline [40 40] & 1 & 0,6436 & 0,8939 & 0,9045 \\
\hline [50 50] & 1 & 0,5832 & 0,4898 & 0,7363 \\
\hline [50 40] & 1 & 0,7646 & 0,7053 & 0,8361 \\
\hline$\left[\begin{array}{ll}50 & 30\end{array}\right]$ & 1 & 0,6385 & 0,3361 & 0,8292 \\
\hline [50 20] & 1 & 0,3835 & 0,7535 & 0,7994 \\
\hline$\left[\begin{array}{ll}50 & 10\end{array}\right]$ & 1 & 0,8847 & 0,9712 & 0,9573 \\
\hline$\left[\begin{array}{lll}50 & 05\end{array}\right]$ & 1 & 0,9323 & 0,8107 & 0,9496 \\
\hline
\end{tabular}

*La arquitectura de la RNA descrito como $\left[k_{1} k_{2}\right]$ se refiere al número de neuronas en las dos capas ocultas, respectivamente

\subsection{EVALUACIÓN DEL DESEMPEÑO DE LA RNA}

El desempeño de una RNA se puede evaluar, usando diversas técnicas [101], entre las cuales la raíz del error promedio cuadrático, RMSE (Root Mean Square Error); el error cuadrático total, SSE (Sum Square Error); el error relativo promedio, MER (Mean Error Ratio); el error promedio cuadrático, MSE (Mean Square Error); y el factor de correlación $R^{2}\left(R^{2}\right.$ Correlation Factor). En el presente trabajo, se diseñó la preparación de datos para utilizar como indicador de desempeño el SSE, mientras que para las fases de aprendizaje (entrenamiento y prueba), y validación de la RNA se usaron el MSE y el factor $R^{2}$; adicionalmente, para la simulación del conjunto total de datos, se usó el factor de correlación $R^{2}$ que es reportado en este documento. Los indicadores mencionados fueron calculados, usando las ecuaciones 5, 6 y 7, así: 


$$
\begin{array}{r}
\text { SEE }=\sum_{t=1}^{T}\left(O_{t}-\bar{O}_{t}\right)^{2} \\
M S E=\sum_{t=1}^{T}\left(\frac{Y_{t}-O_{t}}{T}\right)^{2} \\
R^{2}=1-\frac{\sum_{t=1}^{T}\left(Y_{t}-\bar{O}_{t}\right)^{2}}{\sum_{t=1}^{T}\left(Y_{t}-\bar{O}_{t}\right)^{2}}
\end{array}
$$

Donde, $Y_{t}$ es la salida deseada, $O_{t}$ es la salida obtenida, $\bar{O}_{t}$ es el promedio de las salidas obtenidas y $T$ es el número de registros tomados en cada fase (aprendizaje y validación), y en la simulación con el total de la base de datos.

\subsection{CONFORMACIÓN DE LAS RNA}

En esta investigación, se elaboraron y se entrenaron tres redes neuronales artificiales, con tipología multicapa conformada por una de entrada, una primera oculta con 50 neuronas, una segunda oculta con 10 neuronas, y una de salida con una única neurona correspondiente al valor de la resistencia de diseño a la compresión. Las variables (neuronas), de entrada y salida en cada red neuronal artificial elaborada y entrenada, se muestran en la Tabla 4.

Tabla 4. Conformación de las RNA para la estimación de la resistencia de diseño a la compresión

\begin{tabular}{|c|l|c|}
\hline RNA & \multicolumn{1}{|c|}{ ENTRADAS } & SALIDA \\
\hline RNA_1 & C, C-i, SF, FA, GBFS, W, HWWR, HWWR-i, S, S-i, G, G-i, G-TMA, LTCA-i, AEA, SFR, SFR-i, Lf, Lf/Df & $f^{\prime}$ C \\
\hline RNA_2 & C, C-i, SF, FA, GBFS, W, HWWR, HWWR-i, S, S-i, G, G-i, G-TMA, AEA, SFR, SFR-i, Lf, Lf/Df & $f^{\prime} C$ \\
\hline RNA_3 & C, C-i, SF, FA, GBFS, W, HWWR, HWWR-i, S, S-i, G, G-I, AEA, SFR, SFR-i, Lf, Lf/Df & f'C $^{\prime}$ \\
\hline
\end{tabular}

\section{RESULTADOS Y ANÁLISIS}

Las tres redes neuronales artificiales para estimación de la resistencia de diseño a la compresión en concretos reforzados con fibras de acero, fueron elaboradas y entrenadas, siguiendo el procedimiento descrito en esta investigación. Para las redes elaboradas, se analizó el comportamiento computacional de los datos en las fases de aprendizaje lentrenamiento y pruebal), y de validación. En la Tabla 5, se reporta el factor de correlación $R^{2}$ tanto para las fases mencionadas como para la simulación con la base de registros completa. 
Tabla 5. Comportamiento computacional de las datos en las fases de entrenamiento (aprendizaje y prueba) y de validación, así como de la base de registros completa, para tres redes neuronales artificiales para estimar la resistencia a la compresión en concretos reforzados con fibras de acero

\begin{tabular}{|l|c|c|c|c|}
\hline $\begin{array}{c}\text { FASE } \\
\text { RNA }\end{array}$ & $\begin{array}{c}\text { ENTRENAMIENTO } \\
\mathbf{R}^{\mathbf{2}}\end{array}$ & $\begin{array}{c}\text { PRUEBA } \\
\mathbf{R}^{\mathbf{2}}\end{array}$ & $\begin{array}{c}\text { VALIDACIÓN } \\
\mathbf{R}^{\mathbf{2}}\end{array}$ & $\begin{array}{c}\text { TOTAL DATOS } \\
\mathbf{R}^{\mathbf{2}}\end{array}$ \\
\hline RNA_1 & 1 & 0,88197 & 0,89393 & 0,95785 \\
\hline RNA 2 & 0,99997 & 0,76503 & 0,70385 & 0,84966 \\
\hline RNA 3 & 1 & 0,76895 & 0,94576 & 0,94980 \\
\hline
\end{tabular}

En la Figura 3, para la fase de validación se muestra la correspondencia entre los valores reportados y estimados de la resistencia a la compresión. En el análisis de la Figura 3, se aprecia lo siguiente:

a. RNA

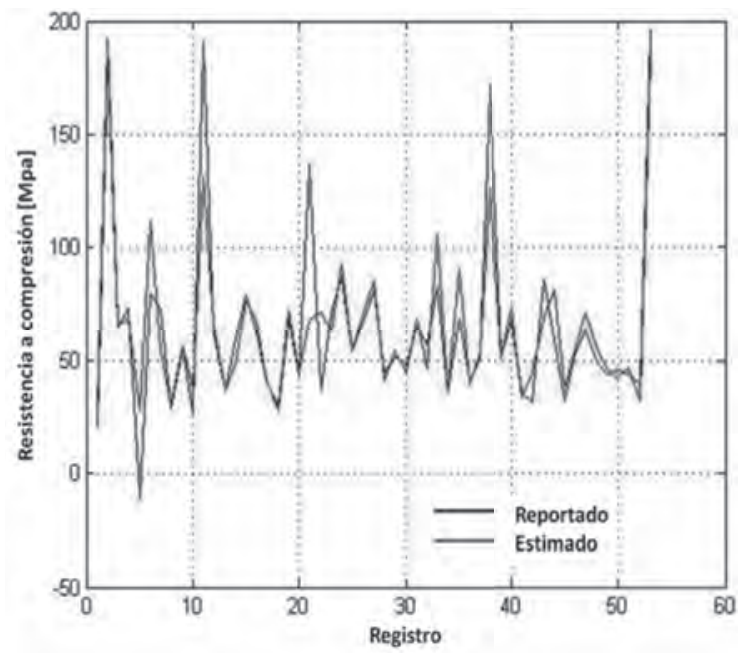

b. RNA 2

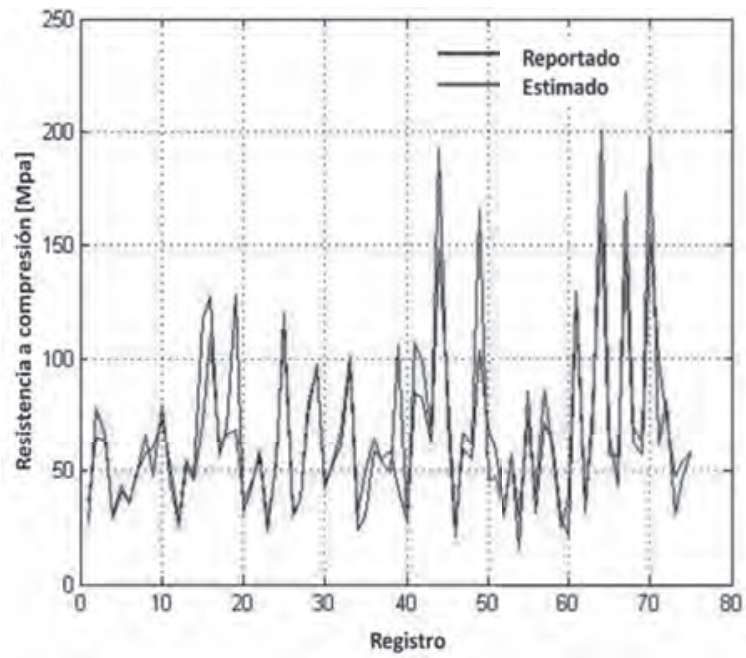


C. RNA 3

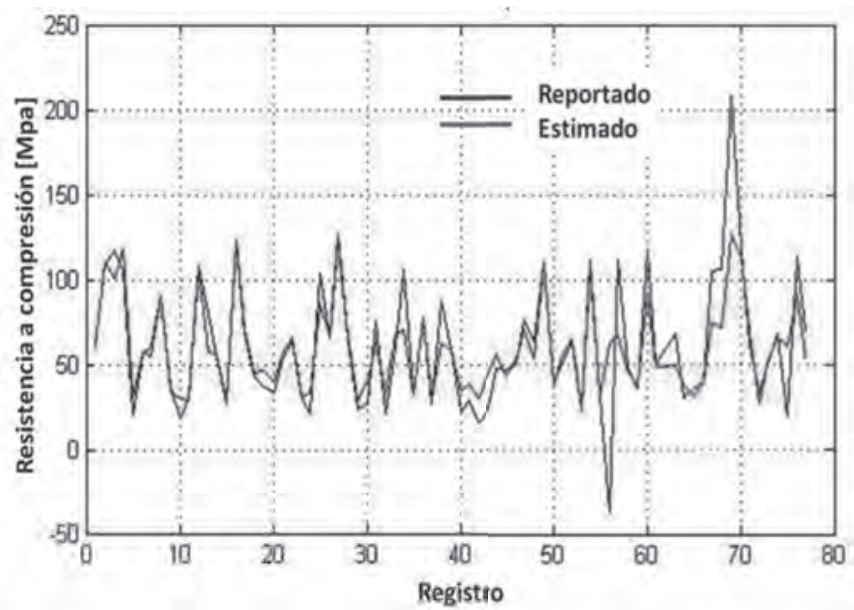

Figura 3. Comportamiento en la fase de validación computacional de las Redes Neuronales Artificiales, entrenadas para estimar la resistencia a la compresión en mezclas de concreto reforzadas con fibras de acero.

Para las tres redes neuronales artificiales, hay un comportamiento equivalente de tendencia de los valores. Se puede notar que para diseños de mezclas conducentes a concretos de resistencia normal de 25-30 MPa, y para diseños de mezclas conducentes a concretos de resistencia intermedia de 30-40 MPa, las RNA logran hacer una adecuada estimación.

Para diseños de mezclas conducentes a concretos de alta resistencia, la estimación en algunos casos es adecuada. Es importante resaltar que algunas características especiales de las mezclas reales (como ambientes y curados controlados, y características intrínsecas en adiciones minerales, en especial las cenizas volantes), no logran ser reconocidas por las RNA. Sin embargo, se puede observar que hasta valores de resistencia a la compresión de 100 MPa, el comportamiento de estimación es aceptable.

En algunos casos muy puntuales (aproximadamente el 2\% de los casos o menos), las RNA son susceptibles de entregar valores negativos de resistencia en la estimación. Se puede notar que la segunda RNA es más robusta para este tipo de situaciones. Este problema se debe principalmente, al modelo y a las redes neuronales artificiales utilizadas, y que puede ser solucionado en versiones futuras mejoradas de la RNA. A pesar de que estas estimaciones con valores negativos constituyen resultados erróneos y excepcionales, no plantean mayores problemas para esta etapa exploratoria de la investigación.

Los resultados en términos generales, muestran un buen comportamiento de estimación en las tres redes neuronales artificiales elaboradas, y es significativo que para la red RNA_l, los indicadores de evaluación sean adecuados, y la estimación de la resistencia a la compresión se haga a partir de la mayor consideración de características en las variables de entrada que es concordante con la teoría sobre el diseño de mezclas de concreto [1], donde un alto número de variables relacionadas con los materiales usados, influyen en el valor de dicha propiedad mecánica. 


\section{CONCLUSIONES}

La utilización de redes neuronales artificiales fue hecha para predecir la resistencia a la compresión en concretos reforzados con fibras de acero. Las redes neuronales artificiales con tipología feedforward, con aprendizaje backpropagation y arquitectura multicapa fueron elaboradas y evaluadas, usando como indicadores de desempeño el error MSEy el factor de correlación $R^{2}$ en la fase de validación. De acuerdo con los indicadores de desempeño mencionados, en términos generales, las redes tienen un buen comportamiento para realizar dicha estimación, siendo significativa una amplia consideración de características en las variables de entradas. El trabajo realizado y los resultados obtenidos, permiten presentar los siguientes aportes:

La consideración de variables de entrada que consisten en diferenciar el tipo de cemento, la procedencia del agregado (triturado, canto rodado), el perfil litológico del agregado grueso, el tipo de agente reductor de agua (plastificante, superplastificante), y el tipo de fibra metálica, adicionales a las dosificaciones de sus componentes (incluidas adiciones minerales), hacen de la RNA una herramienta apropiada de predicción universal de la resistencia a la compresión del concreto con fibras de acero.

La inclusión de registros sin adición de fibra, hacen que la herramienta de predicción pueda usarse también, para estimar la resistencia a la compresión en concretos no reforzados con fibras. Se abre así, una agenda futura de investigación en la tecnología del concreto reforzado con fibra de acero en:

- Realizar ajustes en la RNA para predicción de la resistencia a la compresión, de tal forma que se obtengan mejores indicadores de desempeño.

- Usar la RNA como una herramienta de optimización de los componentes por dosificar en una mezcla de concreto, mediante la evaluación de la variación de la resistencia a la compresión, considerando la variación en las cantidades de un componente en particular.

- Usar la RNA como herramienta de optimización en el volumen de la fibra de acero como refuerzo de la matriz cementicia, mediante la evaluación de la variación de la resistencia a la compresión, considerando no sólo la variación de la cantidad sino también, la morfología y relación de aspecto de la fibra.

\section{REFERENCIAS BIBLIOGRÁFICAS}

[1] Sánchez de Guzmán D., (2000). Tecnología del concreto y del mortero. Bhandar Editores, Bogotá, 349 p.

[2] Mehta P.K., and Monteiro P.J., (2001). Concrete: Microstructure, Properties and Materials. University of California at Berkeley. Berkeley, 239 p. 
[3] Armenta J., Olague C., y Castañeda J., (2004). Influencia de la geometría y concentración de fibras de acero en la tenacidad de un concreto masivo. En: Sendero/Centro T² Chihuahua (1): pp. 3-16.

[4] Ahmed S.F.U., and Mihashi H., (2007). A review on durability properties of strain hardening fibre reinforced cementitious composites (SHFRCC). In: Cement \& Concrete Composites. Vol. 29 (5), pp. 365-376.

[5] Popovics S., (1998). History of a mathematical model for strength development of Portland cement concrete. In: ACI Materials Journal. Vol. 95 (5), pp. 593-600.

[6] Wang J.Z., Ni H.G., and He Y.J., (1999). The application of automatic acquisition of knowledge to mix design of concrete. In: Cement and Concrete Research. Vol. 29 (12), pp. 1875-1880.

[7] Yeh I.C., (1999). Design of high-performance concrete mixture using neural networks and nonlinear programming. In: Journal of Computing in Civil Engineering. Vol. 13 (1), pp. 36-42.

[8] Nath U.K., and Barua P.B., (2004). Optimization of concrete mixture proportioning using Taguchi's method. In: The Indian Concrete Journal. Vol. 78 (9), pp. 52-56.

[9] Ponce C.P., (2010). Inteligencia artificial con aplicaciones a la ingeniería. Alfaomega, México.

[10] López J.A., y Caicedo E., (2006). Una aproximación práctica a las redes neuronales artificiales. Conferencias. Curso de Redes Neuronales Artificiales, Universidad del Valle, Cali, Colombia.

[1 1] Oh J.W., Lee G.W., and Lee I.W., (1997). The Proportioning of Concrete Mixture using Artificial Neural Networks. Seventh International Conference on Computing in Civil and Building Engineering. August 19-21, Seoul, Korea, pp. 1057-1512.

[12] Garg R., (2003). Concrete Mix Design using Artificial Neural Network. Patalia, India, June. Thesis (M. Eng. Civil Eng.), Thapar Institute of Engineering \& Techonology, Deemed University.

[13] Wang J.Z., Wang X.J., and Ni H.G. (2003). An Algorithm of Neural Network and Application to Data Processing in Concrete Engineering. In: Informatica. Vol. 14 (1), pp. 95-110.

[14] Alshihri M.M., Azmy A.M., and El-Bisy M.S., (2009). Neural Networks for predicting compressive strength of structural light weight concrete. In: Construction and Building Materials. Vol. 23, pp. 2214-2219.

[15] Yeh I.C., (2009). Optimization of concrete mix proportioning using a flattened simplex-centroid mixture design and neural networks. In: Engineering with Computers. Vol. 25, pp. 179-190. 
[16] Alilou V.K., and Teshnehlab M., (2010). Prediction of 28-day compressive strength of concrete on the third day using artificial neural networks. In: International Journal of Engineering. Vol. 3 (6), pp. 565-576.

[17] Abdeen M.A., and Hodhod H., (2011). Analytic Formulae for Concrete Mix Design Based on Experimental Data Base and Predicting the Concrete Behavior using ANN Technique. In: International Journal of Engineering. Vol. 4 (5), pp. 368-386.

[18] Kurkova V., (1992). Kolmogorov theorem and multilayer neural networks. IEEE. ASSP Magazine. Vol. 1, pp. 4-22.

[19] Haykin S., (1999). Neural networks: a comprehensive foundation. $2^{\text {nd }}$ edition. Prentice Hall.

[20] Leshno M., Lin V., Pinkus A., and Schocken S., (1993). Multilayer feedforward networks with nonpolynomial activation function can approximate any function. Neural Networks. Vol. 6, pp. 861-867.

[21] Hecht-Nielsen R., (1987). Kolmogorov's mapping neural network existence theorem. Proceedings of The International Conference on Neural Networks. Vol. 3, pp. 11-13, IEEE Press.

[22] Rumelhart D.E., Hinton G.E., and Williams R.J., (1986). Learning internal representations by error propagation. D.E. Rumelhart y J.L. McClelland (eds.). Parallel distributed processing, 1. MIT Press, Cambridge.

[23] Masters T., (1993). Practical Neural Networks recipes in C++. Academic Press, pp. 173-180.

[24] Tabares H., Branch J., y Valencia J., (2006). Generación dinámica de la topología de una red neuronal artificial del tipo Perceptrón multicapa. En: Revista Facultad de Ingeniería. 38, pp. 146-162.

[25] Hinton G., (1989). Connectionist learning procedures. University of Toronto.

[26] Moller M., (1993). Efficient training of feed-forward neural networks. Thesis (PhD). Arhus University. Daimi, Iran.

[27] Lourakis M.I.A., (2005). A brief description or the Levenberg-Mardquardt Algorithm implemented by levmar. Institute of Computer Science, Foundation for Research and Technology - Hellas (FORTH), Heraklion, Greece, February, 6 p.

[28] Diaz W., Will A., y González S.L., (2011). Un método para estimación de calidad de datos en redes neuronales y aplicación a problemas de hormigón de alto performance. XIX Congreso sobre Métodos Numéricos y sus Aplicaciones - ENIEF 2011 . Asociación Argentina 
de Mecánica Computacional (AMCA). Rosario, Argentina, noviembre 1-4. En: Müller O., Signorelli J.W., Storti M.A., (Edit), Mecánica Computacional. Vol. 30 (43), pp. 3381-3381.

[29] Noguchi T., Maruyama I.M., and Kanematsu M., (2003). Performance based desing for concrete mixture with multi-optimizing Genetic Algorithm. In: The $11^{\text {th }}$ International Congress on the Chemistry of Cement (ICCC): Cement's contribution to the development in the $21^{\text {st }}$ Century, Durban, South Africa, pp.11-16, pp.1921-1930.

[30] Alsayed S.H., and Amjad M.A., (1996). Strength, water absorption and porosity of concrete incorporating natural and crushed aggregate. In: Construction and Building Materials. Vol. 23 (6), pp. 109-120.

[31] Cetin A., and Carrasquillo R.L., (1998). High-Performance Concrete: Influence of Coarse Aggregates on Mechanical Properties. In: ACI Materials Journal. Vol. 95 (3), pp. 252-261.

[32] Chan Yam J.L., Solís Carcaño R., y Moreno E.I., (2003). Influencia de los agregados pétreos en las características del concreto. En: Ingeniería, Vol. 7 (2), pp. 39-46.

[33] Mills J.C., and loannides, A.M., (2007). Laboratory study of larger sized aggregate in Portland cement concrete. In: International Journal of Pavement Engineering, Vol. 8 (4), pp. 253-263.

[34] Tsai C.T., Li L.S., Chang C.C., and Hwang C.L., (2009). Durability design and application of steel fiber reinforced concrete in Taiwan. In: The Arabian Journal for Science and Engineering, Vol. 34 (1B), pp. 57-79.

[35] ACl Committee 544, (2002).

[36] Holschemacher K., and Müller T., (2007). Influence of fibre type on hardened properties of steel fibre reinforced concrete. Modern Building Materials, Structures and Techniques. $9^{\text {th }}$ Conference International, May 16-18. Vilnius, Lithuania. En: http://leidykla.vgtu.It/ conferences/MBM_2007/1pdf/Holsemak.pdf (enero de 2012).

[37] Pujadas Álvarez P., (2008). Durabilidad del hormigón con fibras de polipropileno. Trabajo de Grado. Universitat Politécnica de Catalunya. Barcelona. 200 p.

[38] Pandor D.A., (1994). Behavior of high strength fiber reinforced concrete beams in shear, Thesis (M.Sc.). Massachusetts Institute of Technology, Boston. 124 p.

[39] Barros J.A.O., and Figueiras J.A., (1996). Experimental and numerical analysis of steel fiber reinforced concrete slabs. En: I Conferencia Internacional sobre los Materiales Compuestos Aplicados en Arquitectura y Construcción. España. En: http://www.civil.uminho.pt/ composites/Publications/1999/CP1996_001_ICNTSE.pdf (enero de 2012). 
[40] Lie T.T., and Kodur V.R., (1996). Thermal and mechanical properties of steel-fibre-reinforced concrete at elevated temperatures. In: Canadian Journal of Civil Engineering, Vol. 23 (4), pp. 511-517.

[41] Alsayed S.H., and Al-Hozaimy A.M., (1999). Ductility of concrete beams reinforced with FRP bars and steel fibers. In: Journal of Composite Materials. Vol. 33 (19), pp. 1792-1806.

[42] Ay L., (1999). Using prestressed steel fiber reinforced high performance concrete in the industrialization of bridge structures; Licentiate Thesis, Royal Institute of Technology. Stockholm, Sweden. 141 p.

[43] Gustafsson J., and Noghabai K., (1999). Steel fibers as shear reinforcement in high strength concrete beams. En: http://mww.tekna.no/ikbViewer/Content/738968/doc-22-3.pdf (enero de 2012).

[44] Zhand J., Stang H., and Li V.C., (1999). Fatigue life prediction of fiber reinforced concrete under flexural load. In: International Journal of Fatigue. Vol. 21, pp. 1033-1049.

[45] Domingo A., Lázaro C., and Serna P., (2000). Use of steel fiber reinforced concrete in thin shell structures: evaluation of fiber performance through testing of shell specimens. Computational Methods for Shell and Spatial Structures, IASS - IACM 2000, Chania-Crete, Greece, 18 p.

[46] Groth P., (2000). Fibre reinforced concrete; Doctoral Thesis, Luleå Tekniska Universitet. Luleå, Sweden, $237 \mathrm{p}$.

[47] Koiman A.G., Van der Veen C., and Walraven J.C., (2000). Modelling the post-cracking behavior of steel fibre reinforced concrete for structural design purposes. In: HERON, Vol. 45 (4), pp. 275-307.

[48] Zhand J., Stang H., y Li V.C., (2001). Crack bridging model for fibre reinforced concrete under fatigue tension. In: International Journal of Fatigue. Vol. 23, pp. 655-670.

[49] Balendran R.V., Zhou F.P., Nadeem A., and Leung A.Y.T., (2002). Influence of steel fibres on strength and ductility of normal and light-weight high strength concrete. In: Building and Environment. Vol. 37, pp. 1361-1367.

[50] Barragan B.E., (2002). Failure and toughness of steel fiber reinforced reinforced concrete under tension and shear. Thesis (Doctor). Universitat Politecnica de Catalunya, Barcelona.

[51] Gencoglu M., and Eren I., (2002). An experimental study on the effect of steel fiber reinforced concrete on the behavior of the exterior beam-column joints subjected to reversal cyclic loading. In: Turkish J. Eng. Env. Sci. Vol. 26, pp. 493-502. 
[52] Kwak, Y.K., Eberhard, M.O., Kim, W.S., and Kim, J. (2002). Shear strength of steel fiber-reinforced concrete beams without stirrups. In: ACI Structural Journal, July-August, pp.530-538.

[53] Kan Y.C., Pei K.C., and Yang H.C., (2003). An investigation on toughness of steel fiber reinforced heavy concrete. Transactions of the $17^{\text {th }}$ International Conference on Structural Mechanics in Reactor Technology, Prague, Czech Republic, 8 p.

[54] Miao B., Chern J.C., and Yang C.A., (2003). Influences of fiber content on properties of selfcompacting steel fiber reinforced concrete. In: Journal of the Chinese Institute of Engineers. Vol. 26 (4), pp. 523-530.

[55] Barros, J., Pereira, E., Ribeiro, A., Cunha, V., and Atunes, J. (2004). Self-compacting steel fibre reinforced concrete for precasted sandwich panels - experimental and numerical research. Workshop of 6th International RILEM Symposium on fibre reinforced concrete - BEFIB 2004. En: http://www.civil.uminho.pt/composites/Publications/2004/CP2004_002_BeFib2004.pdf (enero de 2012).

[56] Poon C.S., Shui Z.H., and Lam L., (2004). Compressive behavior of fiber reinforced highperformance concrete subjected to elevate temperatures. In: Cement and Concrete Research. vol. 34, pp. 2215-2222.

[57] Yurtseven A., (2004). Determination of mechanical properties of hybrid fiber reinforced concrete. Thesis (MSc.), Middle East Technical University. 82 p.

[58] Neves R.D., and Fernandes de Almeida J.C.O., (2005). Compressive behaviour of steel fibre reinforced concrete. In: Structural Concrete. Vol. 6 (1), pp. 1-8.

[59] Löfgren I., (2005). Fibre reinforced concrete for industrial construction - a facture mechanics approach to material testing and structural analysis, Thesis (PhD). Chalmers University of Technology. Göteborg, Sweden, 276 p.

[60] Löfgren I., Olesen J.F., and Flansbjer M., (2005). Application of WST-Method for fracture testing of fibre-reinforced concrete. In: NT Technical Report, TR-575, Nordic Innovation Centre, Oslo, Norway, 66 p.

[61] Poh J., Tan K.H., Peterson G.L., and Wen D., (2005). Structural testing of steel fibre reinforced concrete (SFRC) tunnel lining segments in Singapore. Report. 17 p.

[62] Sharif M.S.H., (2005). Resistance of plain and fibre-reinforced high-strength concrete to projectile impact. Thesis (M. Eng), National University of Singapore. 125 p. 
[63] Seow P.E.C., (2005). A unified failure criterion for normal, high-strength and steel fibrereinforced concrete. Thesis (PhD), National University of Singapore. 210 p.

[64] Carlsward J., (2006). Shrinkage cracking of steel fibre reinforced self compacting concrete overlays. Test methods and theoretical modeling; Doctoral Thesis, Luleå Tekniska Universitet. Luleå, Sweden, 261 p.

[65] Oucief H., Habita M.F., and Redjel B., (2006). Hybrid fiber reinforced self-compacting concrete: hardened properties. In: International Journal of Civil Engineering, Vol. 4 (2), pp. 77-85.

[66] Sorelli, L.G., Meda, A., and Plizzari, G.A. (2006). Steel fiber concrete slabs on ground: a structural matter. In: ACI Structural Journal, July-August, pp. 551-558.

[67] Caruso C., Hoffman N., Hsu T.T.C., and Mo Y.L., (2007). Constitutive models of prestressed steel-fiber concrete. Report. National Science Foundation, Award EEC-0649163, 16 p.

[68] El Semelawy M., (2007). Effects of axial prestress on the punching behaviour of plain and fibre reinforced concrete slabs. Thesis (M. Applied Sc.), University of Toronto. 191 p.

[69] Ganesan N., Indira P.V., and Abraham R., (2007). Steel fibre reinforced high performance concrete beam-column joints subjected to cycling loading. ISET In: Journal of Earthquake Technology, Technical Note. Vol. 44 (3-4), pp. 445-456.

[70] Kown S.H., Ferron R.P., Akkaya Y., and Shah S.P., (2007). Cracking of fiber-reinforced selfcompacting concrete due to restrained shrinkage. In: International Journal of Concrete Structures and Materials. Vol. 1 (1), pp. 3-9.

[71] De Hanai J.B., and Holanda K.M.A., (2008). Similarities between punching and shear stength of steel fiber reinforced concrete (SFRC) slabs and beams. In: Revista IBRACOM de Estructuras e Materais. Vol. 1 (1), pp. 1-16.

[72] Eggers J., and Rupnow T.D., (2008). Flexural strength and fatigue of steel fiber reinforced concrete (2004 Hale Boggs Deck), Louisiana Transportation Research Center, LTRC Project 05-5C. Baton Rouge, USA. December, 37 p.

[73] Eswari S., Raghunath P.N., and Suguna K., (2008). Ductility performance of concrete with Dramix steel micro-reinforcement. In: Advances in Natural and Applied Sciences. Vol. 2 (3), pp. 243-248.

[74] Eswari S., Raghunath P.N., and Suguna K., (2008). Ductility performance hybrid fiber reinforced concrete. In: American Journal of Applied Sciences. Vol. 5 (9), pp. 1257-1262. 
[75] Fugii A.P., (2008). Avaliação de tubos de concreto reforçados com fibras de aço segundo a NBR 8890; Ilha Solteira, Dissertação (M. Eng. Civil), Universidade Estadual Paulista Julio de Mesquita Filho. Brasil, 163 p.

[76] Kelleher T., (2008). Softening coefficients for prestressed steel fiber reinforced concrete. Report. University of Houston. 20 p.

[77] Singh S.P., Ambedkar B.R., Mohammadi Y., and Kaushik S.K., (2008). Flexural fatigue strength prediction of steel fibre reinforced concrete beams. In: Electronic Journal of Structural Engineering. Vol. 8, pp. 46-54.

[78] Carmona S., y Cabrera M., (2009). Comportamiento al corte de hormigones reforzado con fibras de acero. En: Revista Ingeniería de Construcción. Vol. 24 (1), pp. 79-94.

[79] Flores C.A., (2009). Performance of large scale steel fiber reinforced concrete deep beam with single opening under monotonic loading. Thesis (MSc.), The University of Texas at Arlington. $120 \mathrm{p}$.

[80] Graeff Â.G., Pilakoutas K., Lynsdale C., and Neocleous K. (2009). Corrosion durability steel fibre reinforced concrete. In: Intersections/Intersectii. Vol. 6 (4), pp. 77-89.

[81] Heeralal M., Kumar P.R., and Rao Y.V., (2009). Flexural fatigue characteristic of steel fiber reinforced recycled aggregate concrete (SFRRAC). In: Facta Universitatis. Vol. 7 (1), pp. 19-33.

[82] Perumal P., and Elangovan G., (2009). Correlation of experimental and theoretical strength of superplasticised steel fibre reinforced concrete. In: International Journal of Engineering Studies. Vol. 1 (2), pp. 139-148.

[83] Stengel T., (2009). Fracture toughness of steel fibre reinforced ultra high performance concrete. $7^{\text {th }}$ EUROMECH Solid Mechanics Conference. September 7-11. Lisbon, Portugal, 18 p.

[84] Behbahani H.P., (2010). Flexural behavior of steel fiber reinforced concrete beams. Thesis (MSc.), Universiti Technologi Malaysia. 69 p.

[85] Da Cunha V.M.D.C.F., (2010). Steel fibre reinforced self-compacting concrete (from micromechanics to composite behavior); Thesis (Doctor), Universidade do Minho. Azurém, Portugal. 365 p.

[86] Eren Ö., and Marar K., (2010). Effect of steel fibers on plastic shrinkage cracking of normal and high strength concretes. In: Materials Research. Vol. 13 (2), pp. 135-141. 
[87] Hong K.N., Kang S.T., Kim S.W., Park J.J., and Han S.H., (2010). Material properties of aircured ultra-high-performance steel-fiber-reinforced concrete at early ages. In: International Journal of the Physical Sciences. Vol. 5 (17), pp. 2622-2634.

[88] Nguyen-Minh L., Rovňák M., Tran-Quoc T., and Nguyen-Kim K., (2010). Punching shear resistance of steel fiber reinforced concrete flat slabs. Report. Project T-KTXD-2010-18, Ho Chi Minh University of Technology, University of Security Management of Slovak. Bekaert, Indonesia. 8 p.

[89] Rao B.K., and Ravindra V., (2010). Steel fiber reinforced self-compacting concrete incorporating class F fly ash. In: International Journal of Engineering Science and Technology. Vol. 2 (9), pp. 4936-4943.

[90] Sahoo D.R., and Chao S.H., (2010). Use of fiber reinforced concrete for enhanced performance of deep beams with large openings. Structures Congress. pp. 1981-1990.

[91] Vengatachalapathy $V_{\text {., }}$ and Ilangovan R., (2010). A study on steel fibre reinforced concrete deep beams with and without openings. In: International Journal of Civil and Structural Engineering. Vol. 1 (3), pp. 509-517.

[92] Yusof M.A., Norazman N.M., Ariffin I., Zain F.M., Risby M.S., and Ng C.P., (2010). Normal strength steel fiber reinforced concrete subjected to explosive loading. In: International Journal of Sustainable Construction Engineering \& Technology. Vol. 1 (2), pp. 127-136.

[93] Zai S.A.K., Prasad G.L.E., Gupta N.K., Munidrappa N., and Muthumani K., (2010). Impact behavior of SBR-latex modified fiber reinforced concrete beams. Proceedings of the IMPLAST 2010 Conference. Providence, USA. pp. 12-14.

[94] Abbas A., (2011). Management of steel solid waste generated from lathes as fiber reinforced concrete. In: European Journal of Scientific Research. Vol. 50 (4), pp. 481-485.

[95] Huang B., Cao J., Chen X., and Shu X., (2006). Laboratory and analytical study of permeability and strength properties of previous concrete. National Ready Mix Concrete Association, Concrete Technology Forum: Focus on Pervious Concrete, Nashville, USA. pp. 24-25.

[96] Tomosawa F., Noguchi T., and Onoyama K., (1990). Investigation of fundamental mechanical properties of high-strength concrete. Summaries of Technical Papers of Annual Meeting of Architectural Institute Japan, Tokyo, Japan. pp. 497-498.

[97] Tomosawa F., and Noguchi T., (1993). Relationship between compressive strength and modulus of elasticity of high-strength concrete. Proceedings of the Third International 
Symposium on Utilization of High-Strength Concrete, Lillehammer, Norway. Vol. 2, pp. 12471254.

[98] Nemati K.M., Gardoni P., and Noguchi T., (2008). On modulus of elasticity of high-strength concrete. International Conference on Construction and Building Technology - ICCBT. Vol. C (14), pp. 159-172.

[99] Noguchi T., Tomosawa F., Nemati K.M., Chiaia B.M., and Fantilli A.P., (2009). A practical equation for elastic modulus of concrete. In: ACI Structural Journal. Vol. 106 (5), pp. 690-696.

[100] Beale M.H., Hagan M.T., and Demuth H.B., (2000). Neural Networks Toolbox: User's Guide. Version 4.0, The Math Works Inc., Natick, USA. 404 p. 
